\title{
Induced pluripotent stem cells generated from diabetic patients with mitochondrial DNA A3243G mutation
}

\author{
J. Fujikura $\cdot$ K. Nakao $\cdot$ M. Sone $\cdot$ M. Noguchi $\cdot$ \\ E. Mori $\cdot$ M. Naito $\cdot$ D. Taura $\cdot$ M. Harada-Shiba $\cdot$ \\ I. Kishimoto • A. Watanabe $\cdot$ I. Asaka $\cdot$ K. Hosoda • \\ K. Nakao
}

Received: 4 December 2011 / Accepted: 30 January 2012 / Published online: 7 March 2012

(C) Springer-Verlag 2012

\begin{abstract}
Aims/hypothesis The aim of this study was to generate induced pluripotent stem (iPS) cells from patients with mitochondrial DNA (mtDNA) mutation.

Methods Skin biopsies were obtained from two diabetic patients with mtDNA A3243G mutation. The fibroblasts thus obtained were infected with retroviruses encoding OCT4 (also known as POU5F1), SOX2, c-MYC (also known as MYC) and $K L F 4$. The stem cell characteristics were investigated and the mtDNA mutation frequencies evaluated by Invader assay.
\end{abstract}

Junji Fujikura and Kazuhiro Nakao contributed equally to this study.

Electronic supplementary material The online version of this article (doi:10.1007/s00125-012-2508-2) contains peer-reviewed but unedited supplementary material, which is available to authorised users.

J. Fujikura $(\bowtie) \cdot$ K. Nakao $\cdot$ M. Sone $\cdot$ M. Noguchi $\cdot$ E. Mori $•$

M. Naito $\cdot$ D. Taura $\cdot$ K. Nakao $(\bowtie)$

Department of Medicine and Clinical Science,

Kyoto University Graduate School of Medicine,

54 Shogoin Kawahara-cho, Sakyo-ku,

Kyoto 606-8507, Japan

e-mail: j-fuji@sannet.ne.jp

e-mail:nakao@kuhp.kyoto-u.ac.jp

M. Harada-Shiba $\cdot$ I. Kishimoto

Department of Endocrinology and Metabolism,

National Cerebral and Cardiovascular Center,

Osaka, Japan

A. Watanabe $\cdot$ I. Asaka

Center for iPS Cell Research and Application (CiRA),

Institute for Integrated Cell-Material Sciences,

Kyoto, Japan

K. Hosoda

Department of Human Health Science,

Kyoto University Graduate School of Medicine,

Kyoto, Japan
Results From the two diabetic patients we isolated four and ten putative mitochondrial disease-specific iPS (Mt-iPS) clones, respectively. Mt-iPS cells were cytogenetically normal and positive for alkaline phosphatase activity, with the pluripotent stem cell markers being detectable by immunocytochemistry. The cytosine guanine dinucleotide islands in the promoter regions of OCT4 and NANOG were highly unmethylated, indicating epigenetic reprogramming to pluripotency. Mt-iPS clones were able to differentiate into derivatives of all three germ layers in vitro and in vivo. The Mt-iPS cells exhibited a bimodal degree of mutation heteroplasmy. The mutation frequencies decreased to an undetectable level in six of 14 clones, while the others showed several-fold increases in mutation frequencies (51-87\%) compared with those in the original fibroblasts (18-24\%). During serial cell culture passage and after differentiation, no recurrence of the mutation or no significant changes in the levels of heteroplasmy were seen.

Conclusions/interpretation iPS cells were successfully generated from patients with the mtDNA A3243G mutation. Mutation-rich, stable Mt-iPS cells may be a suitable source of cells for human mitochondrial disease modelling in vitro. Mutation-free iPS cells could provide an unlimited, diseasefree supply of cells for autologous transplantation therapy.

Keywords Gene therapy - Monogenic forms of diabetes . Stem cells

$\begin{array}{ll}\text { Abbreviations } \\ \text { EB } & \text { Embryoid body } \\ \text { ES } & \text { Embryonic stem } \\ \text { FOXA2 } & \text { Forkhead box A2 } \\ \text { iPS } & \text { Induced pluripotent stem } \\ \text { MELAS } & \begin{array}{l}\text { Mitochondrial encephalopathy lactic acidosis } \\ \text { and stroke-like episodes }\end{array}\end{array}$




$\begin{array}{ll}\text { MERRF } & \text { Myoclonic epilepsy and ragged-red fibres } \\ \text { Mt1 } & \text { Mt-iPS patient 1 } \\ \text { Mt2 } & \text { Mt-iPS patient 2 } \\ \text { mtDNA } & \text { Mitochondrial DNA } \\ \text { Mt-iPS } & \text { Mitochondrial disease-specific iPS } \\ \text { NANOG } & \text { Nanog homeobox } \\ \text { SCID } & \text { Severe combined immunodeficient } \\ \alpha \text {-SMA } & \alpha \text {-Smooth muscle actin } \\ \text { SOX } & \text { SRY (sex determining region Y)-box } \\ \text { SSEA } & \text { Stage-specific embryonic antigen } \\ \text { TRA } & \text { Tumour rejection antigen }\end{array}$

\section{Introduction}

Mitochondrial DNA (mtDNA) is present inside mitochondria and codes for components essential for cellular energy production [1]. mtDNA mutations cause degenerative human diseases. The tRNA (Leu) A3243G mutation is one of the most frequently observed mutations of mtDNA and is associated with a wide range of clinical phenotypes, including diabetes mellitus, hearing loss, cardiomyopathy, and mitochondrial encephalopathy, lactic acidosis and stroke-like episodes (MELAS) [2].

The mode of inheritance of mitochondrial diseases is maternal, but the penetrance of the disease is variable [3]. It is not possible to predict the phenotypes of children on the basis of the mother's genotypes and phenotypes, because the segregation of mtDNA tends to follow a pattern of random genetic drift [3]. This is the case for somatic cells and germ cells: it is not possible to predict to which cell types mutant mtDNA will dominantly migrate during development. To date, there is no specific therapy or cure for mitochondrial diseases, only supportive treatment. Efforts to understand the underlying genetics and pathophysiology of mitochondrial diseases have been hampered by the lack of a disease model.

Recently, human induced pluripotent stem (iPS) cells were successfully induced from adult skin fibroblasts [4]. iPS cells are biologically indistinguishable from embryonic stem (ES) cells. Human iPS cells, like ES cells, can differentiate into a variety of cell types and may therefore be another cell source for regenerative medicine. We have previously reported on angiogenic and adipogenic differentiation of human iPS and ES cells [5, 6]. More recently, disease-specific iPS cells have been generated from fibroblasts obtained from patients with various diseases [7-14], although not with mitochondrial diseases. The purpose of the present study was to derive iPS cells from patients with mitochondrial diseases and to evaluate the mtDNA of these cells.

\section{Methods}

Generation of mitochondrial disease-specific iPS cells Skin biopsies were undertaken after informed consent under protocols approved by the Ethics Committee of Kyoto University. Skin samples $(4 \mathrm{~mm})$ were minced with scalpels into smaller pieces and tissue fragments were placed into a tissue culture dish under a sterile coverslip. Medium (DMEM supplemented with $10 \% \mathrm{FBS}$ (wt/vol.) and penicillin/streptomycin; Invitrogen, Carlsbad, CA, USA) was added to completely immerse the coverslip, and dishes were incubated at $37^{\circ} \mathrm{C}$ in a humidified incubator $\left(5 \% \mathrm{CO}_{2}\right)$. Fibroblasts grew out of the tissue fragments and when sufficiently numerous, cells were trypsinised and expanded. Cell cloning by limiting dilution in 96 -well microtitre plates was employed at passage five.

The generation of iPS cells was performed according to the protocol of Ohnuki et al. [15]. In brief, the mouse ecotropic retrovirus receptor Slc7al gene (Addgene, www. addgene.org/) was introduced to patient-derived fibroblasts at around passage number four by infection with lentivirus for $24 \mathrm{~h}$. Retrovirus production was carried out for $24 \mathrm{~h}$ in Plat-E packaging cells via transfection with pMXs-hOCT4, pMXs-hSOX2, pMXs-hKLF4, pMXs-hc-MYC (Addgene) [16]. Fibroblasts expressing the mouse Slc7al gene were then infected with retroviral cocktail. Next day, the medium was replaced with DMEM supplemented with $10 \%$ FBS (wt/vol.). After 6 days of transduction, infected fibroblasts were re-seeded on mouse fibroblast STO cell line feeder cells [17]. The medium was replaced the following day with human ES cell medium (ReproCELL; ReproCELL, Yokohama, Japan) supplemented with $4 \mathrm{ng} / \mathrm{ml}$ basic fibroblast growth factor (Wako, Osaka, Japan) and changed every 2 days. Starting 4 weeks after infection, colonies were picked on the basis of their morphological resemblance to human ES cell colonies and transferred on to mouse fibroblast STO cell line feeder cells in six-well plates; we defined this stage as passage one. Cultures were maintained on mouse fibroblast STO cell line feeders and passaged every 5 to 7 days enzymatically using $0.25 \%$ (wt/vol.) trypsin with $0.1 \mathrm{mg} / \mathrm{ml}$ collagenase type IV. Two human ES cell lines (H9 and KhES-1) and two human iPS cell lines (B7 and G1) were cultured and collected for genomic DNA analysis $[4,18-20]$.

Quantitative assessment of mtDNA mutation frequencies by Invader assay The primary probes and the invader oligo used to detect $\mathrm{A} 3243 \mathrm{G}$ heteroplasmy were as follows: primary probe for 3243A: 5'-CGCGCCGAGGAGCCCGG TAATCGC $<$ amino $>-3$ '; primary probe for $3243 \mathrm{G}$ : 5 '- $\mathrm{ACG}$ GACGCGGAGGGCCCGGTAATCG $<$ amino $>-3$ '; common Invader oligo: 5'-CCCACCCAAGAACAGGGTTTGTTAA GATGGCAGT- $3^{\prime}$. The first 10 and 12 positions in the primary 
probes represent the 5 ' flap of Invader reaction. The cleavage enzyme, fluorescence resonance energy transfer probe, signal probe and Invader oligo were added to the microplates, including diluted plasmids that included the primary probe/ Invader oligo binding region, after which the Invader assay was carried out as previously described [21]. The plates were incubated at $63^{\circ} \mathrm{C}$ in the fluorescence microplate reader (FluoDia-T70; Otsuka Electronics, Osaka, Japan). Fluorescence values for carboxyfluorescein (3243 A; wavelength/ bandwidth: excitation 485/20 nm; emission 530/25 nm) and Redmond red (3243G; excitation 560/20 nm; emission 620/ $40 \mathrm{~nm}$ ) were measured every $2 \min$ for a period of $4 \mathrm{~h}$. To detect $\mathrm{A} 3243 \mathrm{G}$ heteroplasmy, we calculated the copy number of $3243 \mathrm{~A}$ and $3243 \mathrm{G}$ with a standard curve using quantitative Invader assay as described $[21,22]$. The $\mathrm{A} 3243 \mathrm{G}$ ratio was based on the ratio of the all-copy ( $3243 \mathrm{~A}$ and $3243 \mathrm{G}$ ) to the $3243 \mathrm{~A}$ copy. In this assay, the lowest detection limit of the mutation frequency is $2 \%$. The mtDNA A3243G mutation was also analysed by PCR-RFLP or fluorescent correlation spectroscopy [23-25].

Immunocytochemistry and alkaline phosphatase staining Immunocytochemistry was carried out as previously described [26]. The anti-human primary antibodies included: stagespecific embryonic antigen (SSEA)-1, SSEA-3, SSEA-4, tumour rejection antigen (TRA)-1-60, TRA-1-81 (all from Stemgent, San Diego, CA, USA), Nanog homeobox (NANOG) (R\&D Systems, Minneapolis, MN, USA), $\beta 3$ tubulin (Millipore, Temecula, CA, USA), $\alpha$-smooth muscle actin ( $\alpha$-SMA) (Sigma-Aldrich, Saint Louis, MO, USA), forkhead box A2 (FOXA2) (Cell Signaling Technology, Danvers, MA, USA) and SRY (sex determining region Y)box (SOX)17 (R\&D Systems).

For immunofluorescence, Alexa Fluor 488 goat antimouse IgM, Alexa Fluor 488 goat anti-rat IgM, Alexa Fluor 488 goat anti-mouse IgG, Alexa Fluor 546 rabbit anti-goat IgG and Alexa Fluor 546 goat anti-mouse IgG (all from Molecular Probes, Eugene, OR, USA) served as the secondary antibody. Alkaline phosphatase activity was detected using a kit (Alkaline Phosphatase Staining Kit; Stemgent). Images were captured using a microscope (Olympus IX81; Olympus, Tokyo, Japan).

Karyotype analysis Standard G-banding chromosome analysis was performed in the Nihon Gene Research Laboratories (Sendai, Japan) or Chromosome Science Lab (Sapporo, Japan). Selected iPS clones (mitochondrial disease-specific [Mt-iPS] patient 1 [Mt1] clone 1 [Mt1-1], Mt1-4, Mt-iPS patient 2 [Mt2] clone 3 [Mt2-3] and Mt2-6) were analysed at passages 18 to 27 .

Bisulphite genomic sequencing Genomic DNA $(1 \mu \mathrm{g})$ from Mt-iPS cells was processed for bisulphite modification using a kit (EZ DNA Methylation Gold; Zymoresearch, Irvine, CA, USA). The cytosine guanine dinucleotide-rich promoter regions of OCT4 (also known as POU5F1) and $N A N O G$ were selected to be amplified by PCR with ExTaq Hot start (Takara, Kyoto, Japan). The PCR products were subcloned into pCR4 vector (Life Technologies, Carlsbad, CA, USA). Ten clones of each sample were verified by sequencing with Sp6 universal primer.

Short tandem repeat analysis The genomic DNA was used for PCR with Cell ID System (Promega, Madison, WI, USA) and analysed by genetic analyser (ABI PRISM 3100) and GeneMapper version 3.5 (both from Applied Biosystems, Foster City, CA, USA).

In vitro differentiation by embryoid body formation and M15 co-culture Spontaneous differentiation through embryoid body (EB) formation was initiated by dissociation of Mt-iPS cells using collagenase/trypsin treatment, with subsequent transfer to low-attachment multi-well plates in ReproCELL medium. The medium was changed every second day. After 8 days of floating culture, tentative iPS (MtiPS) clones formed EBs and were transferred to $0.1 \%$ (wt/ vol.) gelatin-coated plates to induce further differentiation for 8 days. Differentiated markers such as $\beta 3$-tubulin, $\alpha$ SMA and FOXA2 were analysed by immunocytochemistry.

Endodermal differentiation was performed according to Shiraki et al. [27]. In brief, dissociated Mt-iPS cells were inoculated on to multi-well plates containing a feeder layer of mitomycin C-treated M15 cells [28] in medium (DMEM supplemented with $10 \%$ FBS (wt/vol.) and penicillin/streptomycin; Invitrogen). The medium was changed every second day. After 2 weeks of culture, genomic DNA was extracted for the Invader assay. Endodermal differentiation was confirmed by immunocytochemistry with antibodies against FOXA2 and SOX17.

Teratoma formation Approximately $5 \times 10^{5}$ iPS cells were collected by collagenase/trypsin treatment and injected into the testicles of 7- to 12-week-old severe combined immunodeficient (SCID) mice. Teratomas were collected 9 to 12 weeks after injection and fixed with $10 \%$ (wt/vol.) buffered neutral formalin. Paraffin-embedded tissues were sectioned and stained with haematoxylin and eosin. Animal studies were conducted in accordance with our institutional guidelines and approved by Kyoto University Animal Care Committee.

Determination of mtDNA content Genomic DNA was extracted from blood, fibroblasts and Mt-iPS cells using a kit (DNeasy Tissue Kit; Qiagen, Valencia, CA, USA) or a standard established protocol [29]. The extracted DNA samples were stored at $4{ }^{\circ} \mathrm{C}$ until assay. The relative mtDNA 
copy numbers were measured by real-time PCR and corrected by measurement of the nuclear DNA [30]. The primers for mitochondrial ND5 gene were 5'-AGGCGCTATCAC CACTCTGTTCG-3' and 5'-AACCTGTGAGGAAAGG TATTCCTG-3'. The primers for nuclear $C F$ (also known as CFTR) gene were 5'-AGCAGAGTACCTGAAACAGGAA$3^{\prime}$ and $5^{\prime}$-AGCTTACCCATAGAGGAAACATAA-3'. The PCR was performed using StepOnePlus Real-Time PCR (Applied Biosystems) and a quantitative PCR mix kit (Thunderbird SYBR qPCR Mix; Toyobo, Osaka, Japan). DNA ( $80 \mathrm{ng}$ ) was mixed with $10 \mu \mathrm{l}$ SYBR qPCR containing 6 pmol of forward and reverse primers, and with ROX reference dye in a final volume of $20 \mu \mathrm{l}$. The PCR conditions were 1 min at $95^{\circ} \mathrm{C}$, followed by 40 cycles of denaturation at $95^{\circ} \mathrm{C}$ for $15 \mathrm{~s}$ and of annealing and primer extension at $60^{\circ} \mathrm{C}$ for $60 \mathrm{~s}$. Standard curves were generated using serial dilutions of plasmid DNA containing the PCR amplicons cloned into pGEM-T Easy (Promega). The threshold cycle number values of ND5 and $C F$ were determined in two DNA duplicate samples. The amplified products were denatured and reannealed at different temperature points to detect their specific melting temperature.

Sample mtDNA content (mtDNA copies per cell) were calculated using the formula (ND5 gene copies/CF gene copies $) \times 2=$ mtDNA copies per cell.

\section{Results}

Generation of iPS cells from diabetic patients with the mtDNA A3243G mutation Skin biopsies were obtained from two Japanese patients who had diabetes mellitus, came from different families and carried the mtDNA A3243G mutation. Patient 1 (Mt1) was a 38-year-old man and patient 2 (Mt2) was a 46-year-old woman. Their clinical data are given in Table 1. Mt1 presented at 31 years of age with thirst, polydipsia, polyuria, tiredness and loss of body weight. His blood glucose concentration was $38.4 \mathrm{mmol} / \mathrm{l}$ and his $\mathrm{HbA}_{1 \mathrm{c}}$ level was $14.3 \%$ (132 mmol/mol). He responded well to insulin therapy as a way to control his diabetes. Mt2 was diagnosed with gestational diabetes at 24 years of age. She had a progressive hearing impairment. She had also suffered from epilepsy since the age of 27 years and had been treated with valproic acid. At 31 years of age she developed diabetic ketoacidosis and began insulin therapy. Both patients had a positive family history of maternal diabetes mellitus. The A3243G mtDNA mutation was identified by sequencing of PCR products amplified from peripheral blood genomic DNA from both patients (data not shown).

Two fibroblast lines (Mt1-fibro and Mt2-fibro) were obtained from skin biopsies of the two patients. Each fibroblast line was cultured and infected with a combination of retroviruses encoding the transcription factors octamerbinding protein 4 (OCT4), SOX2, proto-oncogene c-Myc (c-MYC) and Kruppel-like factor 4 (KLF4) [4]. Starting 4 weeks after infection, colonies were selected on the basis of their morphological resemblance to human ES cell colonies and expanded. We were able to isolate four (Mt1-1 to -4 ) and 10 (Mt2-1 to -10) putative iPS (Mt-iPS) clones from Mt1-fibro and Mt2-fibro lines, respectively.

mtDNA mutation frequencies in Mt-iPS cells The presence and levels of heteroplasmy in the patient-derived blood cells, fibroblasts (Mt1-fibro and Mt2-fibro) and putative iPS clones (Mt1-1 to -4 and Mt2-1 to -10 ) were evaluated (Fig. 1a). The Invader assay was used to quantify the heteroplasmy of the mtDNA A3243G mutation in Mt-iPS cells [31-33]. This method was originally developed to genotype single nucleotide polymorphisms and has been used to genotype mtDNA mutations and to quantify heteroplasmy. It is one of the most accurate ways of determining mtDNA heteroplasmy [34]. The passage numbers at which Mt-iPS cells were collected for the Invader assay were: Mt1-1 passage 12; Mt1-2 passage 16; Mt1-3 passage 17; Mt1-4 passage 14; Mt2-1 passage 9; Mt2-2 passage 8; Mt2-3 passage 10; Mt2-4 passage 9; Mt2-5 passage 10; Mt2-6 passage 10; Mt2-7 passage 7; Mt2-8 passage 7; Mt2-9 passage 9; Mt2-10 passage 11. Mutation frequencies in the peripheral blood cells from both patients were 24\% (Mt1blood 24\%, Mt2-blood 24\%). Skin-derived fibroblasts from both patients showed similar levels of mutation frequency compared with those of blood cells from the same patients (Mt1-fibro 18\%, Mt2-fibro 24\%). However, two of four Mt1-iPS clones (Mt1-1 and Mt1-2) and six of 10 Mt2-iPS clones (Mt2-1, Mt2-2, Mt2-3, Mt2-4, Mt2-7, Mt2-8) showed undetectable levels $(<2 \%)$ of the A3243G mutation. Furthermore, marked elevations of the mutation frequencies compared with those of the original fibroblast lines were detected in other iPS lines (Mt1-3 51\%, Mt1-4 87\%, Mt2-5 83\%, Mt2-6 69\%, Mt2-9 79\%, Mt2-10 74\%). No significant associations were found between culture passage number of Mt-iPS cells and the mutation frequency. The absence and presence of the mutation were confirmed by PCR-RFLP and by gene analysis by fluorescence correlation spectroscopy $[24,25]$.

The mutation frequency of the fibroblasts was further assessed (Fig. 1b). The fibroblasts from patient 2 (Mt2fibro) were cultured until passage 16 (for over 2 months). Fibroblast cell cloning by limiting dilution was performed from parental Mt2-fibro at passage five, and five fibroblast clones were obtained (Mt2-fibro-clone). Genomic DNA was extracted from Mt2-fibro at various passage points and from the five Mt2-fibro-clones at about 4 weeks after the cloning procedure. The mutation frequencies in Mt2-fibro gradually increased with increasing passage number of the cells 
Table 1 Information on patient donors for generation of Mt1-iPS and Mt2-iPS cell lines

\begin{tabular}{|c|c|c|c|c|c|c|c|c|c|}
\hline \multirow[t]{2}{*}{ Patient } & \multirow{2}{*}{$\begin{array}{l}\text { Age } \\
\text { (years) }\end{array}$} & \multirow[t]{2}{*}{ Sex } & \multirow[t]{2}{*}{ Family history } & \multirow{2}{*}{$\begin{array}{l}\text { mtDNA } \\
\text { mutation }\end{array}$} & \multirow[t]{2}{*}{ Clinical abnormalities } & \multirow{2}{*}{$\begin{array}{l}\mathrm{BMI} \\
\left(\mathrm{kg} / \mathrm{m}^{2}\right)\end{array}$} & \multirow[t]{2}{*}{ Medication } & \multicolumn{2}{|c|}{$\mathrm{HbA}_{1 \mathrm{c}}(\%)$} \\
\hline & & & & & & & & $\%$ & $\mathrm{mmol} / \mathrm{mol}$ \\
\hline Mt1 & 38 & Male & $\begin{array}{l}\text { Mother: diabetes } \\
\text { mellitus, cardiomyopathy }\end{array}$ & A3243G & Diabetes mellitus & 18 & Insulin $33 \mathrm{U} /$ day & 8.2 & 66.1 \\
\hline Mt2 & 46 & Female & $\begin{array}{l}\text { Mother: diabetes } \\
\text { mellitus }\end{array}$ & A3243G & $\begin{array}{l}\text { Diabetes mellitus, } \\
\text { sensorineural hearing loss, } \\
\text { epilepsy, cardiomyopathy }\end{array}$ & 22 & $\begin{array}{l}\text { Insulin } 30 \mathrm{U} / \text { day, } \\
\text { valproic acid } \\
600 \mathrm{mg} / \text { day }\end{array}$ & 7.0 & 53.0 \\
\hline
\end{tabular}

(Fig. 1b). All the Mt2-fibro-clones displayed high mutation frequencies and no mutation-free fibroblast clones were observed (Fig. 1b).

Characterisation of the Mt-iPS cells generated All the MtiPS clones showed typical human ES cell-like morphology (Fig. 2a, ESM Fig. 1). Mt-iPS cells were positive for
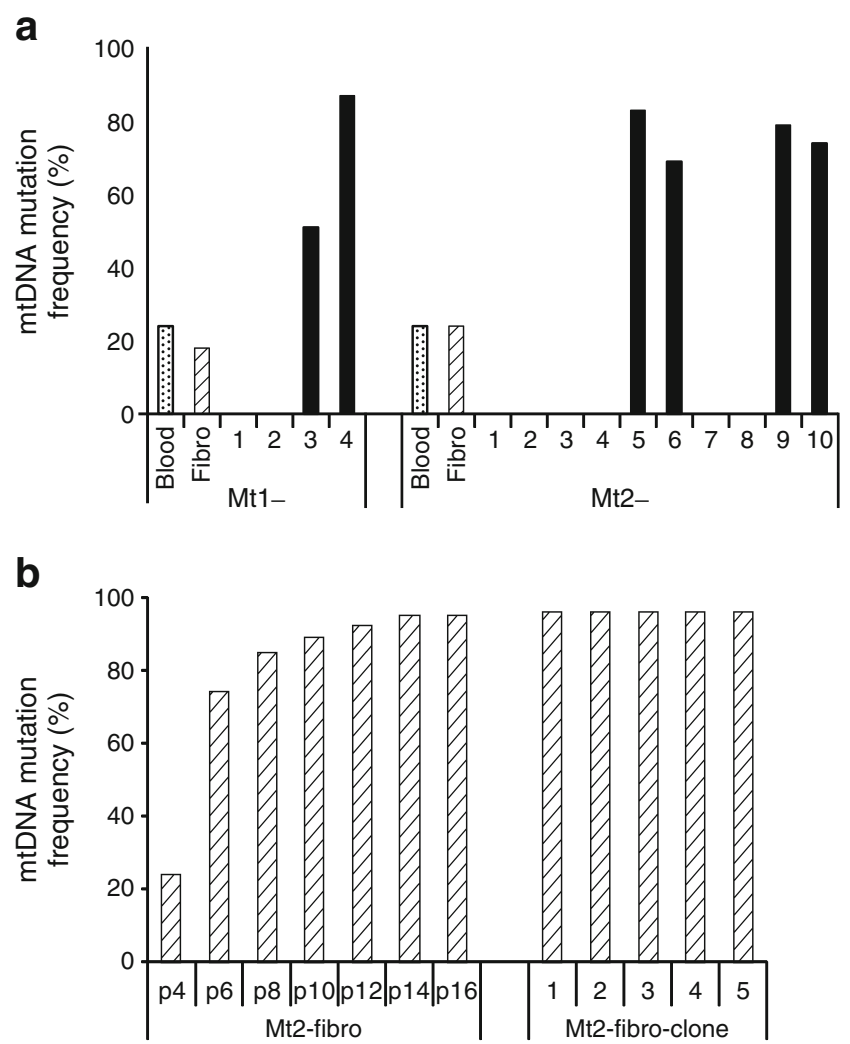

Fig. 1 mtDNA mutation frequencies in Mt-iPS cells. a mtDNA A3243G mutation frequencies in patient-derived blood cells (Mt1blood, Mt2-blood), original fibroblasts (Mt1-fibro, Mt2-fibro) and Mt-iPS clones (Mt1-1 to Mt1-4, Mt2-1 to Mt2-10). Mt-iPS cells were collected at the following passage (p) numbers: Mt1-1 p12, Mt1-2 p16, Mt1-3 p17, Mt1-4 p14, Mt2-1 p9, Mt2-2 p8, Mt2-3 p10, Mt2-4 p9, Mt2-5 p10, Mt2-6 p10, Mt2-7 p7, Mt2-8 p7, Mt2-9 p9, Mt2-10 p11. b mtDNA A3243G mutation frequencies in Mt2-derived fibroblasts at various culture passage numbers and in isolated fibroblast clones. Limiting dilution was performed from the original fibroblasts (Mt2-fibro) at passage five and five fibroblast clones were obtained alkaline phosphatase activity, and the pluripotent stem cell markers SSEA-3, SSEA-4, TRA-1-60, TRA-1-81 and NANOG were detected by immunocytochemical analyses in all 14 clones (Fig. 2a, ESM Fig. 1) [35]. Mt-iPS cells did not produce SSEA-1 except for a few cells at the edge of the colonies (Fig. 2a, ESM Fig. 1). The morphological and immunocytochemical characteristics of mutation-free and mutation-rich Mt-iPS cells were indistinguishable.

To examine whether Mt-iPS clones are cytogenetically normal, karyotyping analyses were performed on selected Mt-iPS cells at passages 18 to 27. Both mutation-free (Mt1-1 and Mt2-3) and mutation-rich (Mt1-4 and Mt2-6) Mt-iPS clones from both patients maintained a normal karyotype (Fig. 2b, ESM Fig. 2).

Bisulphite genomic sequencing analyses evaluating the methylation statuses of cytosine guanine dinucleotides in the promoter regions of OCT4 and NANOG revealed that they were highly unmethylated (Fig. 2c), indicating epigenetic reprogramming to pluripotency.

To confirm that the Mt-iPS clones were indeed derived from the patients, we performed DNA fingerprinting analyses with short tandem repeat markers. The short tandem repeat profiles of the Mt-iPS clones matched perfectly to those of their parental fibroblasts and of blood cells obtained from the patients (ESM Table 1). Thus, the Mt-iPS clones were indeed derived from the patients and were not a result of contamination.

Pluripotency of Mt-iPS cells by in vitro and in vivo differentiation Pluripotent cells are by definition capable of differentiating into cell types derived from each of the three embryonic germ layers [18]. To determine the differentiation ability of Mt-iPS cells in vitro, suspension culture for the formation of EBs was used [36]. After 8 days in suspension culture, iPS cells formed ball-shaped structures. These EBs were transferred to gelatin-coated plates and further cultured for another 8 days. Attached cells showed various types of morphologies, including those resembling neuronal cells, cobblestone-like cells and epithelial cells. Immunocytochemistry detected cells positive for $\beta 3$-tubulin (a marker of ectoderm), $\alpha$-SMA (a marker of mesoderm) or FOXA2 (a marker of endoderm) (Fig. 3a, ESM Fig. 3a). We 
a

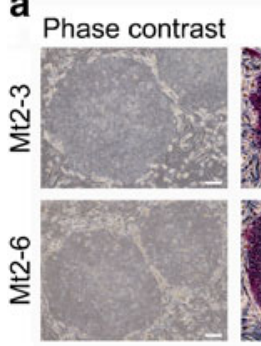

b TRA-1-60

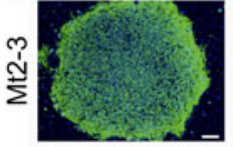

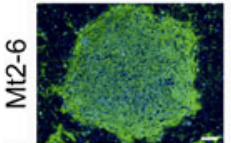
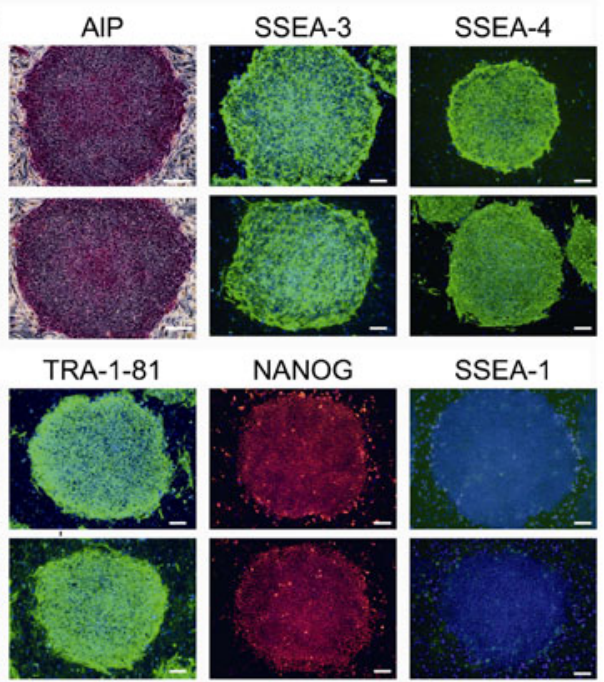

NANOG
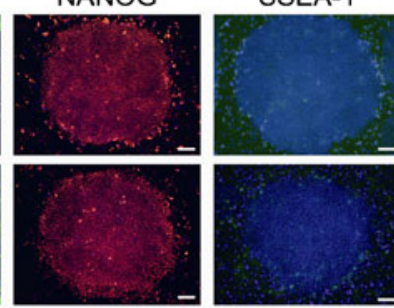

C
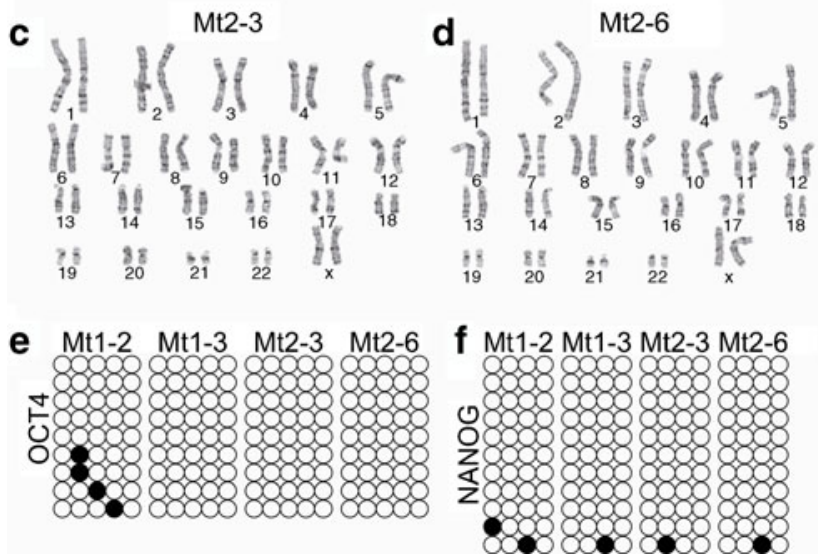

Fig. 2 Characterisation of generated Mt-iPS cells. a, b Colonies of Mt-iPS cells (Mt2-3 and Mt2-6) grown on mouse fibroblast STO cell line feeder cells showing human-ES-cell-like morphology. The detection of (a) alkaline phosphatase activity (AIP) and immunofluorescence analyses for the presence of the pluripotency markers SSEA-3, SSEA-4, and (b) TRA-1-60, TRA-1-81, NANOG and SSEA-1 are indicated. Scale bars $200 \mu \mathrm{m}$. c Karyotyping of Mt-iPS cells Mt2-3 and (d) Mt2-6 at passage 22. e Bisulphite genomic sequencing of the promoter regions of OCT4 and (f) NANOG. White circles, unmethylated cytosine guanine dinucleotides; black circles, methylated cytosine guanine dinucleotides

found that all Mt-iPS clones were able to differentiate into three germ layers in vitro.

To determine pluripotency in vivo, we transplanted MtiPS cells into the testicles of SCID mice. At 9 weeks after injection, tumour formation was observed. Histological examination showed that the tumours contained various tissues, including pigmented epithelium (ectoderm), cartilage (mesoderm) and gut-like epithelial tissues (endoderm) (Fig. 3b, ESM Fig. 3b). Thus, Mt-iPS clones were able to spontaneously differentiate into derivatives of all three germ layers in vivo.
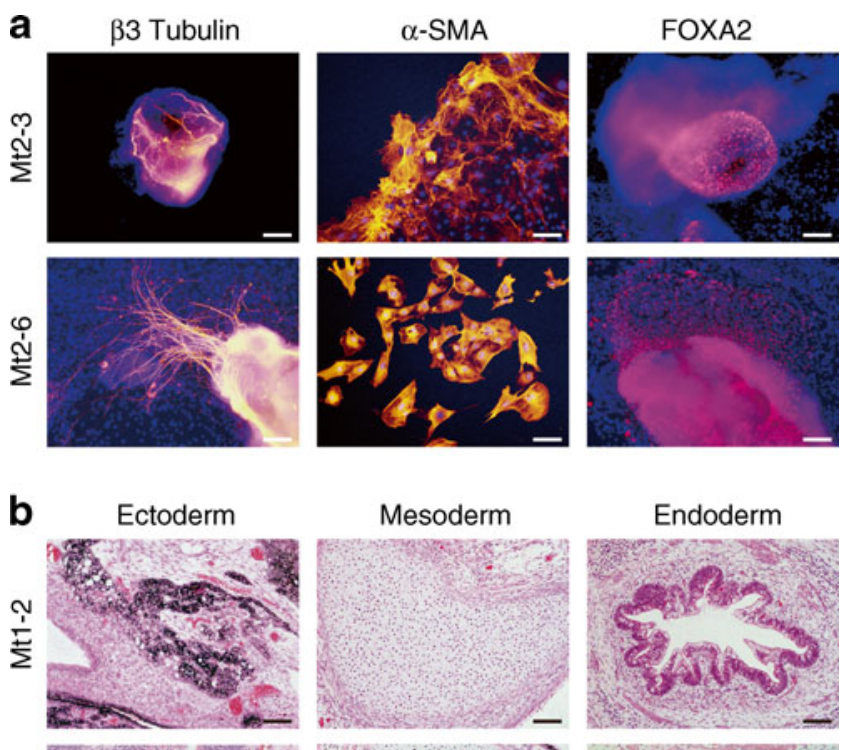

Endoderm
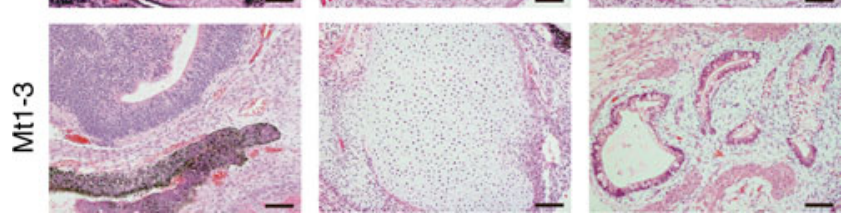

Fig. 3 Pluripotency of Mt-iPS cells by in vitro and in vivo differentiation. a In vitro differentiation of Mt-iPS cells (Mt2-3, Mt2-6) revealed their potential to generate cell derivatives of all three primary germ cell layers. Immunofluorescence analyses showed markers of neurectoderm ( $\beta 3$-tubulin), mesoderm ( $\alpha$-SMA) and endoderm (FOXA2). Images are overlays with a nuclear stain (DAPI). b Teratoma formation occurred after injection of Mt-iPS cells (Mt1-2, Mt1-3) into the testes of SCID mice (Japan Clea, Tokyo, Japan). Haematoxylin and eosin stainings of teratoma sections show derivatives of ectoderm (pigmented epithelial cells), mesoderm (cartilage) and endoderm (gut-like glandular structures). Scale bars, $100 \mu \mathrm{m}$

Influence of culture passage number and differentiation on mtDNA mutation frequencies in Mt-iPS cells We examined whether or not the mutation frequencies of Mt-iPS clones were fixed over the course of cell culturing and passage (Fig. 4a). Analysis of the same clones at various passage numbers revealed that no induction of mutation was observed in mutation-free Mt-iPS clones (Mt1-1, Mt1-2, Mt2-1, Mt2-3 and Mt2-8). Mutation frequencies of the mutation-rich Mt-iPS clones were relatively constant across passages (Mt1-3, Mt1-4, Mt2-6 and Mt2-10).

The influence of differentiation on mutation frequency was also examined in all the Mt-iPS clones (Fig. 4b). Analysis of the same clones in an undifferentiated state and after 16 days of differentiation as a result of spontaneous differentiation (EB formation) or directed differentiation into endodermal lineage (M15 co-culture) (ESM Fig. 4) showed no induction of mutation in mutation-free Mt-iPS clones (Mt1-1, Mt1-2, Mt2-1, Mt2-2, Mt2-3, Mt2-4, Mt2-7 and Mt2-8). The mutation frequencies of the mutation-rich MtiPS clones were relatively constant after differentiation (Mt1-3, Mt1-4, Mt2-5, Mt2-6, Mt2-9 and Mt2-10). 
Fig. 4 Influence of culture passage number and differentiation on mtDNA mutation frequencies in Mt-iPS cells. a mtDNA A3243G mutation frequencies in undifferentiated Mt-iPS clones at various culture passage (p) numbers as indicated. b mtDNA A3243G mutation frequencies in undifferentiated (UD), differentiated $\mathrm{EB}(\mathrm{EB})$ and endodermal (Endo) states of Mt-iPS clones
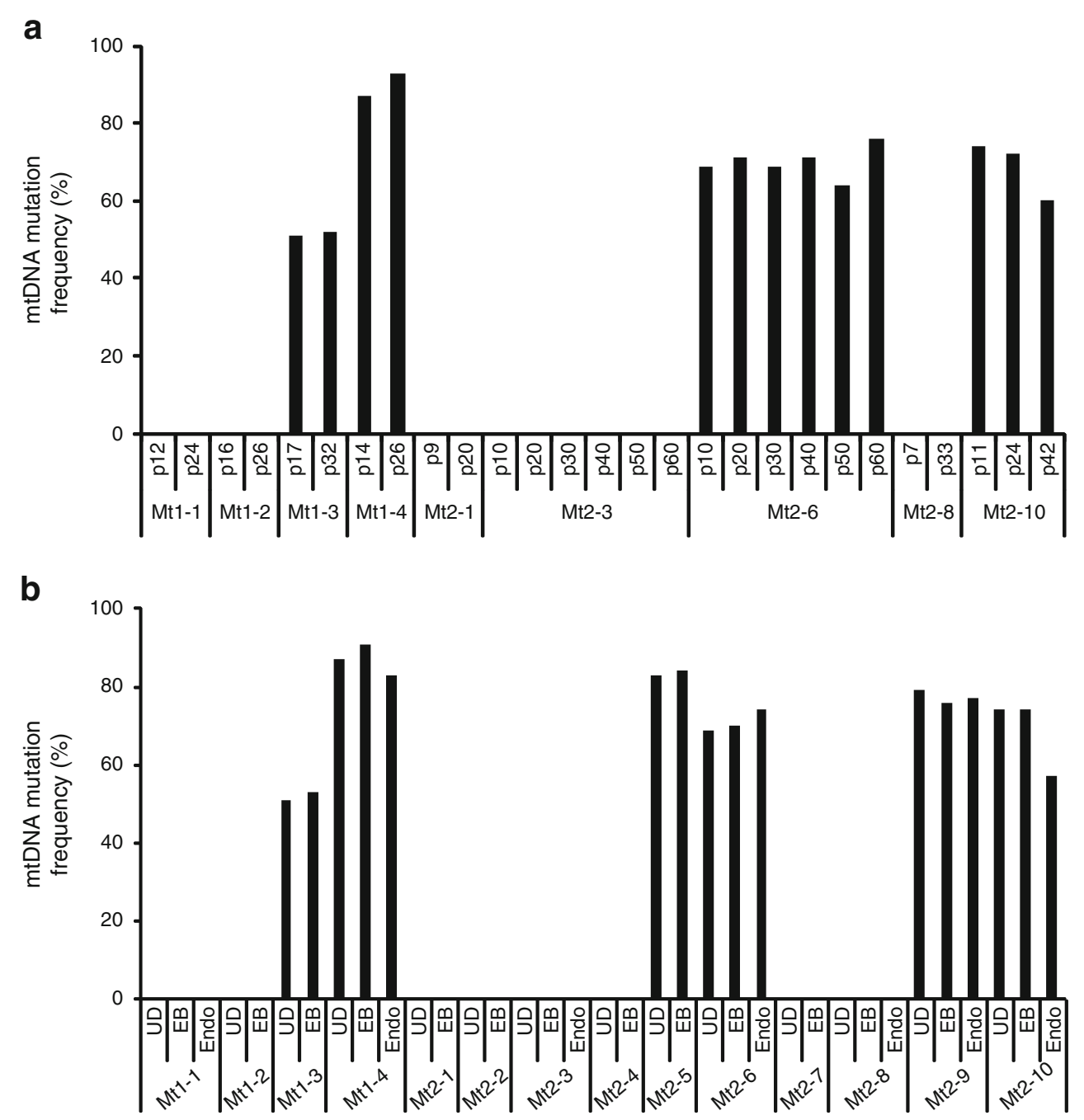

mtDNA content in Mt-iPS cells The mtDNA content (mitochondrial genome copies per cell) in the blood cells, fibroblasts (Mt1-fibro and Mt2-fibro) and iPS clones was determined by quantitative genomic PCR (Fig. 5).

The skin fibroblast mtDNA content (Mt1-fibro 553 copies/cell; Mt2-fibro 4296 copies/cell) was higher than that in the peripheral blood (Mt1-blood 196 copies/cell; Mt2-blood 60 copies/cell). The mtDNA content of Mt-iPS cells at early passage was slightly lower than that in the original fibroblast cultures (Mt1-1 passage 12454 copies/cell; Mt1-4 passage 14151 copies/cell; Mt2-3 passage 10 2,100 copies/cell; Mt2-6 passage 10 1,709 copies/cell) (Fig. 5 and data not shown). The mtDNA content of Mt-iPS cells markedly decreased to levels close to those of human ES (H9 passage 8779 copies/cell; KhES-1 passage 7878 copies/cell) and iPS (B7 passsage 54150 copies/cell; G1 passage 4094 copies/cell) cells by passage number 20 (Mt1-4 passage 26 42 copies/cell; Mt2-3 passage 2049 copies/cell; Mt2-6 passage 2045 copies/cell) and was maintained thereafter (Mt2-3 passage 3068 copies/cell; Mt2-6 passage 3050 copies/cell). The mtDNA content of fibroblasts and iPS cells from Mt1 (Mt1-fibro, Mt1-1 passage 12 and Mt1-4passage 14) were lower than those from Mt2 (Mt2-fibro, Mt2-3

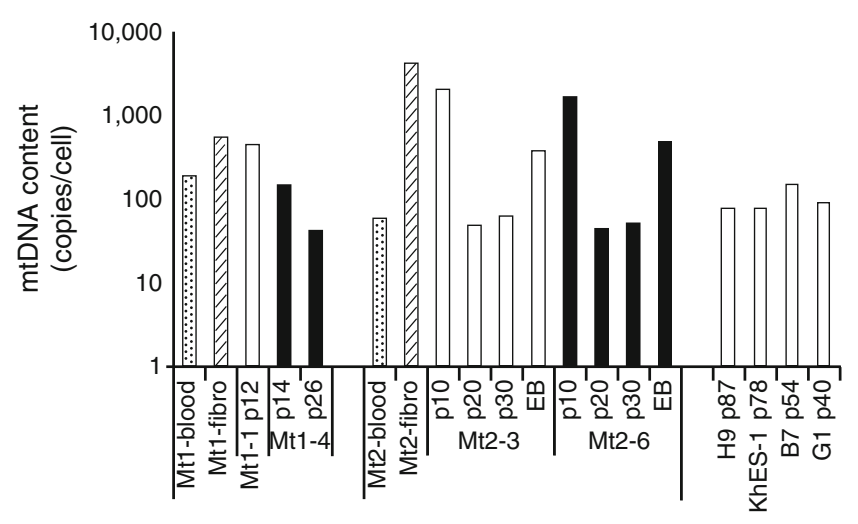

Fig. 5 mtDNA content in Mt-iPS cells. mtDNA content (copies/cell) in blood cells, fibroblasts, mutation-free iPS clones (Mt1-1, Mt2-3 white bars) and mutation-rich iPS clones (black bars). mtDNA from two human ES cell lines (H9 and KhES1) and two human iPS cell lines (B7 and G1) are also shown. p, passage. Mt-iPS cells at around p30 were used for EB formation and assayed for mtDNA contents (Mt2-3 EB, Mt2-6 EB) 
passage 10 and Mt2-6 passage 10), although the content of each Mt-iPS clone was variable (data not shown).

The mtDNA copy number increased seven- to tenfold after EB differentiation (Mt2-3 passage 24-derived EB 384 copies/cell; Mt2-6 passage 24-derived EB 484 copies/cell). Major differences in mtDNA content were not found between mutation-free and mutation-rich iPS cells (Fig. 5 and data not shown).

\section{Discussion}

In the present study, we established human iPS cell lines from male and female diabetic patients with the mtDNA A $3243 \mathrm{G}$ mutation. These cells have the features of pluripotent human ES cells, including the ability to differentiate into cell types of all embryonic lineages.

A striking feature of Mt-iPS shown in the present study is their bimodal levels of heteroplasmy. The mtDNA mutation frequencies decreased to undetectable levels in about half of the clones, while the other half showed a large increase in the levels of mutation heteroplasmy compared with those in the patients' original fibroblasts. The mechanisms underlying this phenomenon remain unclear; however, several possibilities exist in terms of the timing of heteroplasmy segregation. One is that the heteroplasmy levels in Mt-iPS clones simply reflect those in the original single fibroblast from which the iPS clones were derived. This is based on the assumption that the population of fibroblasts is bimodal (mutation-rich and mutation-free) with regard to levels of heteroplasmy, but we were not able to isolate any mutationfree fibroblast clone and hence could find no evidence of extreme mosaicism in the original fibroblasts. The second possibility is that changes in the levels of heteroplasmy occur during serial in vitro culture of Mt-iPS cells. Previous reports have shown age-related directional selection for different mtDNA genotypes in mouse tissues [37]; however, this is unlikely in our study because passage number did not significantly affect the mutation frequencies of Mt-iPS cells. The third and most likely possibility is that changes in the levels of heteroplasmy occur during reprogramming of patients' fibroblasts to iPS cells. Substantial shifts in the levels of mitochondrial heteroplasmy have been demonstrated to occur between single mammalian generations, and neutral mitochondrial genotypes have also been demonstrated to segregate in different directions in offspring from the same female (rapid segregation of mitochondrial genotype) [38-40]. Random partitioning of organelles with few mtDNA molecules into germ cells could account for the small number of segregating units and lead to the rapid segregation of polymorphic mtDNA species in the progeny.

The A3243G transition in the tRNA Leu gene is one of the most frequent mitochondrial mutations [2]. The phenotypic expression of the mutation is variable and may be associated with maternally inherited diabetes mellitus and deafness syndrome, myoclonic epilepsy and ragged-red fibres (MERRF) syndrome, MELAS/MERRF overlap syndrome, maternally inherited Leigh syndrome, and chronic external ophthalmoplegia or Kearns-Sayre syndrome. This heterogeneity is considered to result from the variable levels of heteroplasmy and the variability of tissue-specific thresholds for mitochondrial functions required for normal development and physiology [41]. There is currently no specific therapy or cure. The precise mechanisms for the generation of heteroplasmy and of mitochondrial dysfunction in these diseases remain to be elucidated. Mt-iPS cells offer several significant advantages for this research. The analysis of the process of iPS cell generation might help to clarify the mechanisms of mtDNA germ line segregation. This might further clarify the mode of inheritance of mitochondrial diseases, enabling prefertilisation diagnosis to be performed. We might also be able to study the process of mtDNA somatic segregation toward the target tissues involved in mitochondrial diseases after the induction of differentiation. Mutation-rich Mt-iPS cells should be useful as new types of disease models, in which the initiation and progression of the diseases can be studied. In this context, mutation-free Mt-iPS cells sharing the same nuclear genetic background could serve as ideal negative controls. These approaches could improve understanding of the cause of the disease and lead to the development of efficient preventive and therapeutic strategies. Ultimately, patient-specific and mutation-free Mt-iPS cells might be useful as a supplement or an alternative to disease-affected tissues in future. In this study, we generated Mt-iPS cells by retroviral transduction of OCT4, SOX2, c-MYC (also known as MYC) and KLF4; however, genomic integration of these transgenes increases the risk of tumorigenicity. By generating integrationfree human iPS cells, we could safely transplant mtDNAmutation-corrected cells without the use of potentially harmful DNA recombination technology [42]. mtDNA content is known to be a major determinant of mitochondrial gene expression [43]. Undifferentiated mouse and human ES cells have very low levels of mtDNA content $(<100$ copies/cell), but this rapidly increases up to several thousand-fold during differentiation [44-47]. However, it remains to be determined whether human iPS cells are able, like ES cells, to regulate their mtDNA copy number in their undifferentiated state. We have revealed here that the mtDNA content in Mt-iPS cells at early passage (around passage 10) is similar to that of the original fibroblasts, and that the mtDNA content at later passages (after passage 20) is similar to that of human ES cells. This indicates that the number of mitochondria gradually adapts to the new stem cell environment in iPS cells. Although compensatory amplification of the mitochondrial genome has been reported in patients with mtDNA mutations, the mtDNA content is relatively constant among Mt-iPS 
clones, despite a wide variation in heteroplasmy levels [48-50]. These results also indicate that the cell viability and stemness are unaffected at the low metabolic demand of undifferentiated iPS cells, irrespective of the presence or absence of the A3243G mutation.

In conclusion, we have generated mitochondrial Mt-iPS cells. About half of the clones had undetectable levels of the mutation. By overcoming the immunological and ethical problems associated with ES cells, these Mt-iPS cells could provide a powerful new tool with which to investigate organ involvement and pathogenic mechanisms, and also to screen for new drugs in specific diseases, as well as opening new avenues for autologous cell transplantation therapy.

Acknowledgements We thank K. Nakada and K. Kuwahara for helpful discussions.

Funding This work was supported in part by research grants from: the Leading Project of the Ministry of Education, Culture, Sports, Science and Technology of Japan; the Ministry of Health, Labour and Welfare of Japan; the Takeda Medical Research Foundation; the Smoking Research Foundation; Suzuken Memorial Foundation; Japan Foundation of Applied Enzymology; Novo Nordisk (Insulin Research Award); and Lilly Education and Research Grant Office.

Contribution statement JF, Kazuhiro Nakao, MS, KH and Kazuwa Nakao contributed to study concept and design. JF, Kazuhiro Nakao, MN, EM, MN, DT, MH-S, IK, AW, IA and Kazuwa Nakao contributed to analysis and interpretation of data. JF, Kazuhiro Nakao, MS, MN, EM, MN, DT, MH-S, IK, AW, IA, KH and Kazuwa Nakao contributed to drafting of the manuscript. JF, Kazuhiro Nakao and Kazuwa Nakao contributed to critical revision of the manuscript for intellectual content. All the authors gave approval of the final version to be published.

Duality of interest The authors declare that there is no duality of interest associated with this manuscript.

\section{References}

1. May-Panloup P, Chretien MF, Malthiery Y, Reynier P (2007) Mitochondrial DNA in the oocyte and the developing embryo. Curr Top Dev Biol 77:51-83

2. Finsterer J (2009) Manifestations of the mitochondrial A3243G mutation. Int J Cardiol 137:60-62

3. Shoubridge EA, Wai T (2007) Mitochondrial DNA and the mammalian oocyte. Curr Top Dev Biol 77:87-111

4. Takahashi K, Tanabe K, Ohnuki M et al (2007) Induction of pluripotent stem cells from adult human fibroblasts by defined factors. Cell 131:861-872

5. Sone M, Itoh H, Yamahara K et al (2007) Pathway for differentiation of human embryonic stem cells to vascular cell components and their potential for vascular regeneration. Arterioscler Thromb Vasc Biol 27:2127-2134

6. Taura D, Noguchi M, Sone M et al (2009) Adipogenic differentiation of human induced pluripotent stem cells: comparison with that of human embryonic stem cells. FEBS Lett 583:1029-1033
7. Dimos JT, Rodolfa KT, Niakan KK et al (2008) Induced pluripotent stem cells generated from patients with ALS can be differentiated into motor neurons. Science 321:1218-1221

8. Ebert AD, Yu J, Rose FF Jr et al (2009) Induced pluripotent stem cells from a spinal muscular atrophy patient. Nature 457:277-280

9. Park IH, Arora N, Huo H et al (2008) Disease-specific induced pluripotent stem cells. Cell 134:877-886

10. Soldner F, Hockemeyer D, Beard C et al (2009) Parkinson's disease patient-derived induced pluripotent stem cells free of viral reprogramming factors. Cell 136:964-977

11. Liu J, Verma PJ, Evans-Galea MV et al (2011) Generation of induced pluripotent stem cell lines from Friedreich Ataxia patients. Stem Cell Rev 7:703-713

12. Carvajal-Vergara X, Sevilla A, D'Souza SL et al (2010) Patientspecific induced pluripotent stem-cell-derived models of LEOPARD syndrome. Nature 465:808-812

13. Raya A, Rodriguez-Piza I, Guenechea G et al (2009) Diseasecorrected haematopoietic progenitors from Fanconi anaemia induced pluripotent stem cells. Nature 460:53-59

14. Maehr R, Chen S, Snitow M et al (2009) Generation of pluripotent stem cells from patients with type 1 diabetes. Proc Natl Acad Sci U S A 106:15768-15773

15. Ohnuki M, Takahashi K, Yamanaka S (2009) Generation and characterization of human induced pluripotent stem cells. Curr Protoc Stem Cell Biol Chapter 4: Unit 4A 2

16. Morita S, Kojima T, Kitamura T (2000) Plat-E: an efficient and stable system for transient packaging of retroviruses. Gene Ther 7:1063-1066

17. McMahon AP, Bradley A (1990) The Wnt-1 (int-1) protooncogene is required for development of a large region of the mouse brain. Cell 62:1073-1085

18. Thomson JA, Itskovitz-Eldor J, Shapiro SS et al (1998) Embryonic stem cell lines derived from human blastocysts. Science 282:11451147

19. Fujioka T, Yasuchika K, Nakamura Y, Nakatsuji N, Suemori H (2004) A simple and efficient cryopreservation method for primate embryonic stem cells. Int J Dev Biol 48:1149-1154

20. Nakagawa M, Koyanagi M, Tanabe K et al (2008) Generation of induced pluripotent stem cells without Myc from mouse and human fibroblasts. Nat Biotechnol 26:101-106

21. Yamamoto M, Kakihana K, Ohashi K et al (2009) Serial monitoring of T315I BCR-ABL mutation by Invader assay combined with RT-PCR. Int J Hematol 89:482-488

22. Hall JG, Eis PS, Law SM et al (2000) Sensitive detection of DNA polymorphisms by the serial invasive signal amplification reaction. Proc Natl Acad Sci U S A 97:8272-8277

23. Kadowaki T, Kadowaki H, Mori Y et al (1994) A subtype of diabetes mellitus associated with a mutation of mitochondrial DNA. N Engl J Med 330:962-968

24. Katagiri H, Asano T, Ishihara H et al (1994) Mitochondrial diabetes mellitus: prevalence and clinical characterization of diabetes due to mitochondrial tRNA(Leu(UUR)) gene mutation in Japanese patients. Diabetologia 37:504-510

25. Palo K, Mets U, Jager S, Kask P, Gall K (2000) Fluorescence intensity multiple distributions analysis: concurrent determination of diffusion times and molecular brightness. Biophys J 79:28582866

26. Nagano M, Katagiri S, Takahashi Y (2006) ATP content and maturational/developmental ability of bovine oocytes with various cytoplasmic morphologies. Zygote 14:299-304

27. Shiraki N, Yoshida T, Araki K et al (2008) Guided differentiation of embryonic stem cells into Pdx1-expressing regional-specific definitive endoderm. Stem Cells 26:874-885

28. Larsson SH, Charlieu JP, Miyagawa K et al (1995) Subnuclear localization of WT1 in splicing or transcription factor domains is regulated by alternative splicing. Cell 81:391-401 
29. Laird PW, Zijderveld A, Linders K, Rudnicki MA, Jaenisch R, Berns A (1991) Simplified mammalian DNA isolation procedure. Nucleic Acids Res 19:4293

30. Prigione A, Fauler B, Lurz R, Lehrach H, Adjaye J (2010) The senescence-related mitochondrial/oxidative stress pathway is repressed in human induced pluripotent stem cells. Stem Cells 28:721-733

31. Lyamichev V, Mast AL, Hall JG et al (1999) Polymorphism identification and quantitative detection of genomic DNA by invasive cleavage of oligonucleotide probes. Nat Biotechnol 17:292-296

32. Mashima Y, Nagano M, Funayama T et al (2004) Rapid quantification of the heteroplasmy of mutant mitochondrial DNAs in Leber's hereditary optic neuropathy using the Invader technology. Clin Biochem 37:268-276

33. Inagaki Y, Mashima Y, Fuse N, Ohtake Y, Fujimaki T, Fukuchi T (2006) Mitochondrial DNA mutations with Leber's hereditary optic neuropathy in Japanese patients with open-angle glaucoma. Jpn J Ophthalmol 50:128-134

34. Mazunin IO, Volodko NV, Starikovskaya EB, Sukernik RI (2010) Mitochondrial genome and human mitochondrial diseases. Mol Biol 44:665-681

35. Adewumi O, Aflatoonian B, Ahrlund-Richter L et al (2007) Characterization of human embryonic stem cell lines by the International Stem Cell Initiative. Nat Biotechnol 25:803-816

36. Itskovitz-Eldor J, Schuldiner M, Karsenti D et al (2000) Differentiation of human embryonic stem cells into embryoid bodies compromising the three embryonic germ layers. Mol Med 6:88-95

37. Jenuth JP, Peterson AC, Shoubridge EA (1997) Tissue-specific selection for different mtDNA genotypes in heteroplasmic mice. Nat Genet 16:93-95

38. Ashley MV, Laipis PJ, Hauswirth WW (1989) Rapid segregation of heteroplasmic bovine mitochondria. Nucleic Acids Res 17:73257331

39. Laipis PJ, van de Walle MJ, Hauswirth WW (1988) Unequal partitioning of bovine mitochondrial genotypes among siblings. Proc Natl Acad Sci U S A 85:8107-8110

40. Larsson NG, Tulinius MH, Holme E et al (1992) Segregation and manifestations of the mtDNA tRNA(Lys) A->G(8344) mutation of myoclonus epilepsy and ragged-red fibers (MERRF) syndrome. Am J Hum Genet 51:1201-1212

41. Leonard JV, Schapira AH (2000) Mitochondrial respiratory chain disorders II: neurodegenerative disorders and nuclear gene defects. Lancet 355:389-394

42. Chou BK, Mali P, Huang X et al (2011) Efficient human iPS cell derivation by a non-integrating plasmid from blood cells with unique epigenetic and gene expression signatures. Cell Res 21:518-529

43. Williams RS, Salmons S, Newsholme EA, Kaufman RE, Mellor J (1986) Regulation of nuclear and mitochondrial gene expression by contractile activity in skeletal muscle. J Biol Chem 261:376380

44. Cho YM, Kwon S, Pak YK et al (2006) Dynamic changes in mitochondrial biogenesis and antioxidant enzymes during the spontaneous differentiation of human embryonic stem cells. Biochem Biophys Res Commun 348:1472-1478

45. Facucho-Oliveira JM, Alderson J, Spikings EC, Egginton S, St John JC (2007) Mitochondrial DNA replication during differentiation of murine embryonic stem cells. J Cell Sci 120:4025-4034

46. Harvey A, Gibson T, Lonergan T, Brenner C (2010) Dynamic regulation of mitochondrial function in preimplantation embryos and embryonic stem cells. Mitochondrion 11:829-838

47. St John JC, Facucho-Oliveira J, Jiang Y, Kelly R, Salah R (2010) Mitochondrial DNA transmission, replication and inheritance: a journey from the gamete through the embryo and into offspring and embryonic stem cells. Hum Reprod Update 16:488-509

48. Wong LJ, Perng CL, Hsu CH et al (2003) Compensatory amplification of mtDNA in a patient with a novel deletion/duplication and high mutant load. J Med Genet 40:e125

49. Kim K, Lecordier A, Bowman LH (1995) Both nuclear and mitochondrial cytochrome c oxidase mRNA levels increase dramatically during mouse postnatal development. Biochem J 306:353358

50. Ostronoff LK, Izquierdo JM, Enriquez JA, Montoya J, Cuezva JM (1996) Transient activation of mitochondrial translation regulates the expression of the mitochondrial genome during mammalian mitochondrial differentiation. Biochem J 316:183-191 